\title{
Editorial
}

\section{Peripheral nerve surgery}

\author{
EDWARD R. LAWS, JR., M.D. \\ Department of Neurological Surgery, University of Virginia Health Sciences Center, \\ Charlottesville, Virginia
}

Surgery performed to treat the peripheral and autonomic nervous system has a rich history and an important position in the evolution of modern neurological surgery. Peripheral nerve injuries occur quite often as a result of armed combat. Such injuries have frequently been identified as indications for surgery by military surgeons, particularly during the 19 th century when there was an upsurge in survivable nerve injuries sustained in battle. One recalls that causalgia was first characterized and diagnosed during the US Civil War. As surgery developed in the latter part of the 19th century and the early portion of the 20th century, a number of innovative pioneering general surgeons, some of whom ultimately became neurosurgeons, wrote about the challenging aspects of peripheral and autonomic nervous system surgery. These included two important general surgeons from Chicago, Drs. J. B. Murphy and Alan Kanavel (one of the original members of the Society of Neurological Surgeons). Also included in this group were Drs. C. H. Frazier from the University of Pennsylvania and Alfred Adson and James Learmonth from the Mayo Clinic in Rochester, Minnesota; Adson became very well known for his contributions to surgery of the autonomic nervous system.

World War I stimulated additional interest in peripheral nerve surgery, and Dr. Harvey Cushing and his neurosurgical colleagues began to deal effectively with peripheral nerve injuries by using "modern" techniques. World War II provided even more of an impetus for the development of peripheral nerve surgery and significant contributions were made by Drs. Stookey of New York, Woodhall of Duke University, and Poppen of the Leahey Clinic.

In the 1960s microsurgical concepts and techniques began to be developed. It is interesting to note that some of the most important contributors to microneurosurgery include individuals such as Madjid Samii and Vinko Dolenc, who began their careers as peripheral nerve surgeons.

Clearly not every neurosurgeon is able to concentrate effectively within a relatively narrow field such as peripheral nerve surgery; however, two centers that were developed in North America under the leadership of neurosurgeons made great strides in the field both in practice and research. These centers are the University of Toronto under the guidance of Dr. Alan Hudson and Louisiana State University in New Orleans under the leadership of Dr. David Kline. Some neu- rosurgeons have made peripheral nerve surgery one of their particular areas of expertise; these include Dr. McGillicuddy of the University of Michigan and Dr. Suzie Tindall at Emory University. Other neurosurgeons have followed a different track and have become qualified in more than one specialty that is important to the overall care of patients with peripheral nerve injuries. These include Dr. John Persing of Yale University, who has board certification in neurosurgery and plastic surgery; Dr. Susan MacKinnon, who has appointments in both plastic surgery and neurosurgery at Washington University in St. Louis, Missouri; and Dr. Rob Spinner, who has board certification in both orthopedic surgery and neurosurgery and is responsible for the Peripheral Nerve Program at the Mayo Clinic.

Because peripheral nerve injuries are a constant aspect of the management of trauma, there is an essential tension between community medical facilities, which provide routine care for injured patients, and specialized centers, where expertise from a number of different fields can be brought to bear on particularly complex problems of nerve and brachial plexus injuries. Obviously, the need for decentralized treatment of relatively straightforward peripheral nerve problems will continue; this is one reason why the American Board of Neurological Surgery includes experience in peripheral nerve surgery in its requirements for education and residency experience, as supervised by the Residency Review Committee.

The study presented in this issue emphasizes the nature of peripheral nerve cases managed by neurosurgeons currently in the US. The statistics it provides certainly reflect the competition for complex cases that exists among plastic surgeons, orthopedic surgeons, neurosurgeons, and hand surgeons. This competition may help advance the technical aspects of the field. The cooperation of individuals from these various disciplines is important when one considers the impact of limb reimplantation, vascular microsurgery, tendon transfers, and joint fusions on the surgical treatment of patients with complex peripheral nerve injuries, as well as the need for specialized rehabilitation for these patients. It is clear that neurosurgeons will remain active in the field of peripheral nerve surgery and, perhaps to a lesser extent, in surgery of the autonomic nervous system. One hopes that a few centers will continue to produce the excellent basic 
research that arises from our understanding of the molecular biology of nerve physiology and nerve transmission. We can anticipate that new techniques and new concepts will continue to be developed, and we hope that neurosurgeons will participate in the evolution of this important area of clinical and basic neuroscience.

RESPONSE: I thank Dr. Laws for his cogent and insightful remarks concerning peripheral nerve surgery and the results of my survey. He has correctly pointed out that many past innovations in peripheral nerve surgery were promulgated by neurosurgeons. Unfortunately, this illustrious history contrasts with what the survey reveals. Presently, the majority of neurosurgeons are increasingly uncomfortable with all but the most basic peripheral nerve procedures (that is, open carpal tunnel release). David Kelly once pointed out that, unless neurosurgeons became more comfortable and active with spinal surgery the field would be lost to others; in much the same way peripheral nerve surgery may be lost. The outcome of this survey should serve as a wake-up call to all members of the neurosurgical community. Unless neurosurgeons are better and more fully trained in peripheral nerve surgery the area will be lost to other specialists who feel greater comfort. It would be most unfortunate if peripheral nerve surgery, an area of the nervous system, were lost by neurosurgery to other specialties.

Allen Maniker, M.D. Neurological Institute of New Jersey

Newark, New Jersey 


\title{
Peripheral nerve surgery and neurosurgeons: results of a national survey of practice patterns and attitudes
}

\author{
Allen Maniker, M.D., And Marian Passannante, Ph.D. \\ Department of Neurological Surgery, Neurological Institute of New Jersey, University of Medicine \\ and Dentistry of New Jersey, Newark, New Jersey
}

\begin{abstract}
Object. The goal of this study was to determine current practice patterns and attitudes of neurosurgeons toward peripheral nerve surgery.

Methods. A 13-question survey was mailed to all active members of the American Association of Neurological Surgeons and the Congress of Neurological Surgeons. Collected responses were entered into a database and were analyzed using statistical software.

Conclusions. Of 3800 surveys mailed there were 1728 responses for a $45 \%$ response rate. Analysis of the data revealed that respondents had a greater comfort level with simple peripheral nerve procedures, such as carpal tunnel release, and a lack of comfort with more complex peripheral nerve procedures, such as brachial plexus exploration. The majority of simple cases were treated by the surveyed neurosurgeons, whereas the majority of complex cases were referred to other surgeons, primarily to other neurosurgeons. The type of medical practice (academic, group, or solo) and the location of the practice (major city, small city, suburban setting, or rural area) showed a statistically significant correlation to simple case referral patterns, whereas the length of time since the respondent underwent training did not. Practice type and location, and years since training showed a statistically significant correlation to complex case referral patterns. Only $48.7 \%$ of the respondents believed that they had been given sufficient exposure to peripheral nerve surgery during residency training. The overwhelming majority $(97.2 \%)$ of respondents favored keeping peripheral nerve surgery as part of the neurosurgical curriculum.
\end{abstract}

\section{KEY WORDS • peripheral nerve • survey}

$\mathrm{N}$ EUROLOGICAL surgery has long defined itself as the medical specialty concerned with the diagnosis and treatment of patients with injury to, or diseases of, the brain, spine, and peripheral nerves. When considered separately, no surgeon has ever questioned the appropriateness of diseases of the brain as an integral part of the specialty. In the recent past, however, neurosurgeons and organized neurosurgery have found the need to make a concerted effort to reaffirm their commitment to the spine and spine surgery as a part of the neurosurgical practice. ${ }^{3}$ The same questions can now be asked of neurosurgeons and their relationship with peripheral nerve surgery. A credible, although undocumented, opinion has been that fewer neurosurgeons are now performing surgery on the peripheral nervous system. If this indeed is the case, who is performing peripheral nerve surgery?

Although peripheral nerve surgery is an obvious, logical extension of neurosurgical practice, orthopedic and plastic surgeons have also claimed it as part of their domains. What types of peripheral nerve procedures do neurosurgeons feel

Abbreviations used in this paper: AANS = American Association of Neurological Surgeons; CI = confidence interval; CNS = Congress of Neurological Surgeons; EMG = electromyography; NAP = nerve action potential. comfortable with and still perform? What types do they refer to other surgeons? What factors influence this referral pattern? Is training adequate in this area and should it remain a part of the neurosurgical curriculum? None of these questions has ever been addressed in a systematic, statistically valid, methodologically sound manner.

In an effort to answer these questions and establish an understanding of the practice patterns and attitudes of neurosurgeons, a national survey of all active American members of both major neurosurgical organizations, the AANS and the CNS, was undertaken. The results of this survey are presented.

\section{Clinical Material and Methods}

A 13-question survey was developed to examine the practice patterns and attitudes of active members of the AANS and the CNS (Table 1). Three thousand eight hundred surveys were initially mailed with a return envelope enclosed. Approximately 2 weeks later, an email reminder was sent to all members for whom email addresses were known. Four weeks after the initial mailing, a second mailing with another copy of the survey, an enclosed reminder, and a response envelope was sent to all nonresponders. All 


\section{A. Maniker and M. Passannante}

TABLE 1

\section{Peripheral nerve survey}

1. How comfortable do you feel performing the following procedures? (Please circle the number that corresponds to the following scale for each item.)

SCALE: $1=$ not at all comfortable; $2=$ somewhat uncomfortable; $3=$ somewhat comfortable; 4 = very comfortable; NA = not applicable, don't do.

Carpal tunnel release Open

Endoscopic

Ulnar nerve release Cubital tunnel decompression

Subcutaneous transposition

Submuscular transposition

Suprascapular nerve release

Lateral femoral cutaneous nerve release

Tarsal tunnel release

Brachial plexus exploration for trauma

Peripheral nerve tumor removal

Sural nerve biopsy

Resection of painful neuroma

Nerve repair and grafting

$\begin{array}{lllll}1 & 2 & 3 & 4 & \text { NA }\end{array}$

2. How many of the following procedures do you do per year?

SCALE: $1=$ none; $2=<10$ procedures; $3=10-20$ procedures; $4=>20$ procedures

Carpal tunnel release Open

Endoscopic

Ulnar nerve release

Cubital tunnel decompression

Subcutaneous transposition

Submuscular transposition

Suprascapular nerve release

Lateral femoral cutaneous nerve release

Tarsal tunnel release

Brachial plexus exploration for trauma

Peripheral nerve tumor removal

Sural nerve biopsy

Resection of painful neuroma

Nerve repair and grafting

$\begin{array}{llll}1 & 2 & 3 & 4 \\ 1 & 2 & 3 & 4 \\ 1 & 2 & 3 & 4 \\ 1 & 2 & 3 & 4 \\ 1 & 2 & 3 & 4 \\ 1 & 2 & 3 & 4 \\ 1 & 2 & 3 & 4 \\ 1 & 2 & 3 & 4 \\ 1 & 2 & 3 & 4 \\ 1 & 2 & 3 & 4 \\ 1 & 2 & 3 & 4 \\ 1 & 2 & 3 & 4 \\ 1 & 2 & 3 & 4\end{array}$

3. If you are doing nerve procedures do you use intraoperative (EMG) monitoring? (Please circle the appropriate answer.)
A. yes
B. no
C. NA, don't do nerve procedures

4. If you are doing nerve procedures do you use nerve action potentials (NAP) to evaluate nerve intraoperatively? (Please circle the appropriate answer.)
A. yes
B. no
C. NA, don't do nerve procedures

5. Do you care for the complex peripheral nerve cases (i.e. brachial plexus) yourself or do you refer them out? (Please circle the appropriate answer.)

$\begin{array}{ll}\text { A. } & \text { myself } \\ \text { B. } & \text { refer out }\end{array}$

6. If referred out do they go to: (Please circle the appropriate answer.)
A. A plastic surgeon
B. An orthopedic surgeon
C. Another neurosurgeon

7. Do you care for the simple peripheral nerve cases (i.e. carpal tunnel) yourself or do you refer them out? (Please circle the appropriate answer.)

A. myself

B. refer out
TABLE 1 (continued)

8. If referred out do they go to: (Please circle the appropriate answer.)
A. A plastic surgeon
B. An orthopedic surgeon
C. Another neurosurgeon

9. Would you describe yourself as being in a(n): (Please circle the appropriate answer.)
A. Academic practice
B. Private practice group
C. Solo practice

10. Would you describe your location as: (Please circle the appropriate answer.)
A. Major city
B. Small city
C. Rural
D. Suburban

11. Have you been out of training: (Please circle the appropriate answer.)
A. Less than 5 years
B. 5-9 years
C. $10-14$ years
D. 15 or more

12. Were you given sufficient exposure to peripheral nerve surgery in your training? (Please circle the appropriate answer.)
A. yes

B. no

13. Do you feel that peripheral nerve training should continue to be a part of the neurosurgical resident curriculum? (Please circle the appropriate answer.)

$\begin{array}{ll}\text { A. } & \text { yes } \\ \text { B. no }\end{array}$

active members of the AANS and CNS were sent copies of the survey. Active members were defined for this study as those who resided in the US, were up to date in paying organizational dues, and were not retired or resident members. Any individual who was a member of both organizations was initially mailed only one copy of the survey.

Respondents were asked to rate their comfort level with performing particular peripheral nerve surgical procedures by using a four-point scale response. The respondents were also asked to provide the number of each of these procedures that they performed each year on a graduated scale. The remaining questions provided multiple-choice responses. A final section was included for written comments. The complete survey questions and their format are included in Table 1.

Once the completed surveys were received, the data were entered into a computer running the JMP statistical software package (SAS, Cary, NC) for analysis. Ninety-five percent CIs were generated for prevalence estimates and chi-square and Fisher exact tests were used to identify statistical associations between nominal variables. Nonparametric (Wilcoxon rank sum) tests were used to analyze ordinal data. Statistical testing was conducted at the alpha 0.05 significance level, that is, a probability value lower than 0.05 was considered statistically significant.

\section{Results}

A total of 3800 surveys were mailed. There were 1728 responses, giving us a $45 \%$ response rate. Of the total num- 
TABLE 2

Types of practice

\begin{tabular}{lc}
\hline \hline Practice Type & No. of Respondents $(\%)$ \\
\hline no response* & $9(0.530)$ \\
academic & $430(25.309)$ \\
private group & $871(51.265)$ \\
solo practice & $389(22.896)$ \\
total & $1699(100.000)$ \\
\hline
\end{tabular}

* If no answer was circled on the survey form it was identified as "no response." This applies to all other tables.

ber of responses, 29 were returned from retired members and were therefore excluded from the study results. A total of 1699 responses were determined to be usable.

\section{Characteristics of the Respondents}

Respondents were asked to describe their practice setting and location, and the length of time since they completed their training (Tables 2-4). The majority of respondents $(51.3 \%)$ belonged to private practice groups with the remaining neurosurgeons split almost evenly between academic and solo private practices ( 25.3 and $22.9 \%$, respectively). Forty-nine percent of respondents reported practicing in a major city and $36.8 \%$ in a small city; fewer respondents reported practicing in a suburban or rural area (8.5 and $5.4 \%$, respectively). When asked the length of time since they had undergone training, $47.4 \%$ answered 15 years or more; $18.4 \%, 10$ to 14 years; $17 \%, 5$ to 9 years; and $17 \%$ reported less than 5 years since they had received training.

\section{Respondents and Specific Peripheral Nerve Procedures}

Respondents were asked to rate their comfort level while performing specific peripheral nerve surgical procedures. Table 5 provides the proportion of respondents who were not at all comfortable, somewhat uncomfortable, somewhat comfortable, and very comfortable while performing these procedures. Respondents were also given the opportunity to state that they did not perform the procedure in question.

Respondents reported some variability in their level of comfort according to specific peripheral nerve procedures. Among the most comfortable was open carpal tunnel release, for which greater than $93.5 \%$ of respondents believed they fell into the "somewhat comfortable" or "very comfortable" categories. Neurosurgeons also fell into "comfortable" categories in descending order for sural nerve biopsy $(84.6 \%)$, ulnar nerve cubital tunnel release $(82.8 \%)$, ulnar nerve subcutaneous release $(77.9 \%)$, peripheral nerve tumor removal $(73.7 \%)$, removal of a painful neuroma $(66.6 \%)$, nerve repair and grafting $(58.3 \%)$, and ulnar submuscular transposition (55.6\%). The majority of neurosurgeons felt somewhat uncomfortable or not at all comfortable with lateral femoral cutaneous nerve release (30.4\%), closed endoscopic carpal tunnel release (34\%), tarsal tunnel release $(34.4 \%)$, suprascapular nerve release $(35.3 \%)$, and brachial plexus exploration for trauma (40\%).

Respondents were also asked to specify the number of procedures that they perform each year. Table 6 outlines
TABLE 3

Locations of practice

\begin{tabular}{lc}
\hline \hline Practice Location & No. of Respondents $(\%)$ \\
\hline major city & $833(49.203)$ \\
small city & $625(36.917)$ \\
suburban area & $144(8.506)$ \\
rural area & $91(5.375)$ \\
total & $1693(100.000)$ \\
\hline
\end{tabular}

how the responses were grouped into: none, fewer than 10 procedures, 10 to 20 procedures, and more than 20 procedures.

Some peripheral nerve procedures are more commonly performed than others, with open carpal tunnel release heading the list in frequency. Greater than $27.3 \%$ of respondents reported performing at least 20 open carpal tunnel procedures per year. The proportion of respondents who reported performing more than 20 procedures per year, broken down by procedure were the following in descending order: open carpal tunnel release (27.3\%), ulnar nerve cubital tunnel release $(5.8 \%)$, ulnar nerve subcutaneous release $(3.9 \%)$, endoscopic carpal tunnel release (2.9\%), sural nerve biopsy $(2.2 \%)$, ulnar submuscular release $(1.8 \%)$, nerve repair and grafting (1.4\%), brachial plexus exploration $(0.9 \%)$, tumor removal $(0.6 \%)$, resection of a painful neuroma $(0.6 \%)$, lateral femoral cutaneous nerve release $(0.3 \%)$, tarsal tunnel release $(0.3 \%)$, and suprascapular nerve release $(0.2 \%)$.

A correlation was noted between comfort level and the number of procedures performed. Those neurosurgeons who reported being comfortable or somewhat comfortable with open carpal tunnel release, ulnar nerve cubital tunnel decompression, brachial plexus exploration for trauma, and peripheral nerve tumor removal also reported that they performed more of these procedures than those who reported being not at all comfortable or somewhat uncomfortable $(\mathrm{p}<0.0001)$.

\section{Respondents and use of EMG and NAP Studies}

Respondents were also asked to report on their usage of EMG and NAP studies to evaluate nerves intraoperatively. Only $34 \%$ (95\% CI 31.7-36.2) of respondents reported that they use EMG and an even smaller proportion, 35\% (95\% CI 32.6-37.1) of respondents, reported monitoring NAPs intraoperatively to evaluate nerve function.

There was an association between the number of years spent out of training and the use of both intraoperative

TABLE 4

Time since training

\begin{tabular}{cc}
\hline \hline Time Since Training & No. of Respondents $(\%)$ \\
\hline no response & $4(0.235)$ \\
$<5$ yrs & $288(16.951)$ \\
$5-9$ yrs & $289(17.010)$ \\
$10-14$ yrs & $312(18.364)$ \\
$\geq 15$ yrs & $806(47.440)$ \\
total & $1699(100.000)$ \\
\hline
\end{tabular}




\section{A. Maniker and M. Passannante}

TABLE 5

Responses of 1699 neurosurgeons to the following question: "How comfortable do you feel performing the following procedures?"*

\begin{tabular}{|c|c|c|c|c|c|c|}
\hline \multirow[b]{2}{*}{ Procedure } & \multicolumn{6}{|c|}{$\%$ Responding } \\
\hline & 1 & 2 & 3 & 4 & NA & NR \\
\hline \multicolumn{7}{|l|}{ carpal tunnel release } \\
\hline open & 1.3 & 1.7 & 5.2 & 88.3 & 2.8 & 0.5 \\
\hline endoscopic & 27.8 & 6.2 & 3.6 & 6.8 & 51.7 & 3.8 \\
\hline \multicolumn{7}{|l|}{ ulnar nerve release } \\
\hline cubital tunnel decompression & 3.6 & 3.5 & 9.0 & 73.8 & 8.6 & 1.3 \\
\hline subcutaneous transposition & 4.1 & 5.9 & 11.3 & 66.6 & 10.3 & 1.8 \\
\hline submuscular transposition & 9.3 & 11.7 & 16.0 & 39.6 & 21.3 & 2.1 \\
\hline suprascapular nerve release & 21.5 & 13.8 & 17.8 & 13.0 & 31.5 & 2.3 \\
\hline $\begin{array}{l}\text { lateral femoral cutaneous nerve } \\
\text { release }\end{array}$ & 16.6 & 13.8 & 19.9 & 20.4 & 27.5 & 1.8 \\
\hline tarsal tunnel release & 20.8 & 13.6 & 16.2 & 15.9 & 31.0 & 2.3 \\
\hline $\begin{array}{l}\text { brachial plexus exploration for } \\
\text { trauma }\end{array}$ & 23.2 & 16.8 & 17.7 & 14.1 & 26.2 & 2.0 \\
\hline peripheral nerve tumor removal & 6.5 & 8.0 & 29.2 & 44.5 & 10.2 & 1.5 \\
\hline sural nerve biopsy & 3.3 & 3.7 & 10.4 & 74.2 & 7.6 & 0.8 \\
\hline resection of painful neuroma & 6.5 & 10.7 & 22.5 & 44.1 & 14.2 & 1.9 \\
\hline nerve repair \& grafting & 9.5 & 15.4 & 28.1 & 30.2 & 15.8 & 1.0 \\
\hline
\end{tabular}

* Scaled responses: $1=$ not at all comfortable; $2=$ somewhat uncomfortable; 3 = somewhat comfortable; 4 = very comfortable; NA = not applicable, do not do this; $\mathrm{NR}=$ no response/missing response.

EMG and NAP electrophysiological monitoring modalities. Respondents who were likely to use EMG and NAP monitoring had completed their training more recently than those respondents who did not rely on these techniques (EMG $\mathrm{p}<0.001$; NAP monitoring $\mathrm{p}<0.013$ ).

There was an association between exposure to peripheral nerve surgery during training years and the use of intraoperative EMG and NAP studies. Respondents who reported having been given sufficient exposure to peripheral nerve surgery during training were more likely to use EMG and NAP monitoring and techniques $(\mathrm{EMG}, \mathrm{p}<0.0001$; NAP monitoring $\mathrm{p}<0.0001$ ).

\section{Respondents' Referral Patterns}

A series of questions was asked regarding management and referral patterns for both complex and simple peripheral nerve cases. When asked who cares for patients with complex peripheral nerve problems (such as exploration of the brachial plexus) $76.5 \%$ answered that they refer these patients to other surgeons, whereas $22.1 \%$ care for these patients themselves. When asked to whom they refer these patients, $73 \%$ of respondents answered to other neurosurgeons, $7.9 \%$ to plastic surgeons, and 5\% to orthopedic surgeons.

The pattern of referring complex peripheral nerve cases correlated with practice type: $38.8 \%$ of academic neurosurgeons answered that they would manage these cases themselves, whereas only $17 \%$ of respondents in private group practice and $16 \%$ of those in solo practice responded that they would keep these cases. This was statistically significant $(\mathrm{p}<0.0001)$.

The retention of complex peripheral nerve cases correlated to practice location. Of those respondents who kept complex cases, $60.3 \%$ were located in major cities, $30.7 \%$ in
TABLE 6

Responses of 1699 neurosurgeons to the following question: "How many of the following procedures do you do per year?" *

\begin{tabular}{|c|c|c|c|c|c|}
\hline \multirow[b]{2}{*}{ Procedure } & \multicolumn{5}{|c|}{$\%$ Responding } \\
\hline & 1 & 2 & 3 & 4 & NR \\
\hline \multicolumn{6}{|l|}{ carpal tunnel release } \\
\hline open & 10.2 & 29.8 & 31.1 & 27.3 & 1.5 \\
\hline endoscopic & 79.8 & 4.0 & 1.3 & 2.9 & 11.9 \\
\hline \multicolumn{6}{|l|}{ ulnar nerve release } \\
\hline cubital tunnel decompression & 28.4 & 47.4 & 14.5 & 5.8 & 3.9 \\
\hline subcutaneous transposition & 36.3 & 44.8 & 9.3 & 3.9 & 5.8 \\
\hline submuscular transposition & 59.5 & 27.6 & 3.5 & 1.8 & 7.6 \\
\hline suprascapular nerve release & 77.6 & 17.6 & 0.2 & 0.2 & 4.4 \\
\hline $\begin{array}{l}\text { lateral femoral cutaneous nerve } \\
\text { release }\end{array}$ & 70.0 & 25.5 & 0.6 & 0.3 & 3.5 \\
\hline tarsal tunnel release & 76.6 & 18.9 & 0.4 & 0.3 & 3.9 \\
\hline $\begin{array}{l}\text { brachial plexus exploration for } \\
\text { trauma }\end{array}$ & 69.8 & 23.3 & 2.2 & 0.9 & 3.8 \\
\hline peripheral nerve tumor removal & 38.8 & 54.1 & 3.1 & 0.6 & 3.4 \\
\hline sural nerve biopsy & 33.2 & 52.9 & 8.6 & 2.2 & 3.0 \\
\hline resection of painful neuroma & 55.4 & 39.7 & 0.8 & 0.6 & 3.6 \\
\hline nerve repair \& grafting & 56.2 & 36.8 & 2.6 & 1.4 & 2.9 \\
\hline
\end{tabular}

* Scaled responses: $1=$ none; $2=$ fewer than 10 procedures; $3=10$ to 20 procedures; $4=$ more than 20 procedures; $\mathrm{NR}=$ no response $/$ missing response.

small cities, $4.8 \%$ in suburban settings, and $4.3 \%$ in rural areas. This too was statistically significant $(\mathrm{p}<0.0001)$.

Of those neurosurgeons who retained care of patients with complex peripheral nerve problems, $27.5 \%$ had spent less than 5 years since training; $25.8 \%, 10$ to 14 years; $21.7 \%, 5$ to 9 years; and $19.5 \%$, more than 15 years since training. This was significant $(\mathrm{p}<0.016)$.

When asked who manages simple peripheral nerve cases (that is, carpal tunnel), virtually all neurosurgeons (89.6\%) answered that they manage these cases themselves, whereas $9.5 \%$ refer these cases to other surgeons. When asked to whom they refer these cases, $16.7 \%$ answered to other neurosurgeons, $5.9 \%$ to orthopedic surgeons, and $2.5 \%$ to plastic surgeons.

Of the respondents who refer simple peripheral nerve cases, $43.5 \%$ are academics, $39.1 \%$ are members of private practice groups, and $17.4 \%$ are in solo practice. This was a statistically significant difference $(\mathrm{p}<0.0001)$.

Of the respondents who refer simple cases elsewhere, those in major cities accounted for $65.6 \%$. This percentage was followed by neurosurgeons in small cities $(22.5 \%)$, those in suburban settings (8.1\%), and those in rural areas $(3.8 \%)$. This finding was statistically significant $(\mathrm{p}=$ $0.0001)$.

When management of simple peripheral nerve cases was compared with the number of years since training, no statistically significant difference was noted. Unlike complex cases, all neurosurgeons, regardless of the number of years since their training continue to perform these types of procedures.

\section{Respondents and Training}

When asked if they were given sufficient exposure to peripheral nerve surgery during training, less than half $(48.7 \%)$ of the respondents believed that they had. There 
was an association between the number of years out of training and sufficient exposure to peripheral nerve surgery during training. Those respondents who had undergone training more recently were more likely to believe that they had not been given sufficient exposure to peripheral nerve surgery during training.

Finally, when asked if peripheral nerve training should continue to be a part of the neurosurgical resident curriculum, $97.2 \%$ answered in the affirmative.

\section{Discussion}

This survey was undertaken to assess the attitudes and practice patterns of neurosurgeons toward peripheral nerve surgery as we enter into the 21 st century. The survey was designed to reach as many practicing neurosurgeons in the United States as possible, so that a more accurate picture of current patterns could be formulated.

The response rate to this survey was $45 \%$, which falls within the expected rates for physician surveys. ${ }^{1,2}$ We acknowledge that, given a $45 \%$ response rate, some response bias may have been introduced (that is, the attitudes of the respondents toward peripheral nerve surgery may be different from those of nonresponders). Nevertheless, a comparison of respondent's practice location, practice type, and training variables with pertinent data provided by the AANS census survey (from Calendar Year 2000, the most current year available) suggest that the respondents did provide a representative picture of the group as a whole.

This survey indicates that the vast majority of neurosurgeons refer complex peripheral nerve cases to other surgeons, most often to other neurosurgeons. An increasing comfort level with any particular procedure correlated across the spectrum of difficulty with the increasing number of procedures reported per year (that is, more cases meant more comfort). Academic neurosurgeons as well as those located in major cities are most likely to retain these types of complex cases. The reasons for this most likely are related to the location of large academic centers in major cities, where complex cases are most often referred and retained. The suburban neurosurgeon, who possibly has the easiest access to large academic centers, is least likely to retain these cases. Neurosurgeons in small cities and rural areas may have fewer options for referring patients and, therefore, are more likely to retain these cases than their suburban counterparts.

Neurosurgeons who have just completed training are also most likely to retain management of complex cases, which may be a reflection of their proximity to residency training and, hence, familiarity with techniques, operative procedures, exposures, and so forth. The need for these younger neurosurgeons to establish a practice with retention of all cases presented to them may also influence this decision; however, this is speculation.

Those neurosurgeons who have been in practice longer than 15 years since their training are least likely to retain complex cases. This referral pattern may be due to a lack of comfort with these procedures because of the length of time since training and the rarity of encountering such cases. Furthermore, for the established neurosurgeon and practice group, the need to retain difficult or financially unrewarding cases no longer exists. The latter reason was frequent- ly identified, in the "comments" section of the survey, as the motivation for referral of complex cases. Many comments also reflected a perceived large liability in managing complex peripheral nerve cases with again little financial incentive.

Regarding simple peripheral nerve cases, virtually all neurosurgeons $(89.6 \%)$ treat the cases themselves. Small numbers are referred, again to other neurosurgeons. For simple peripheral nerve cases (in contradistinction to complex cases) academic neurosurgeons are least likely to retain these cases. This finding may be related to the dynamics of academic groups in which superspecialization within the group may be more pronounced, making referral to a colleague's specializing in peripheral nerve surgery more likely, hence a lower rate of case retention. Those neurosurgeons in major cities are also less likely to retain simple peripheral nerve cases in comparison with their small city, suburban, and rural counterparts. This may be attributed to the structure of academic groups, in which even simple cases are handed over to the specialized partner. Interestingly, the performance of simple cases did not correlate with the number of years since training. Across the continuum, from recent neurosurgical resident to established surgeon, all simple cases were retained.

Approximately half of the respondents answered affirmatively that they used EMG and NAP monitoring intraoperatively to assess nerve function. Those who believed that they had received adequate exposure to peripheral nerve surgery during training were more apt to use these methods. This is likely a reflection of the fact that a training program that offered adequate exposure to peripheral nerve surgery was also more likely to incorporate the latest and most sophisticated electrophysiological methods available.

Neurosurgeons in practice for a longer period were identified as being less likely to use these modes of monitoring. The high proportion of neurosurgeons not using EMG and NAP studies unfortunately calls to question the sophistication with which some aspects of peripheral nerve surgery are being undertaken, because these monitoring studies are essential to the proper performance of many procedures.

A troubling finding was that fully one half $(50.2 \%)$ of the respondents believed that they were not given adequate exposure to peripheral nerve surgery during their training. There was a statistical correlation between those who had recently completed training and those who believed they had received inadequate exposure to peripheral nerve surgery. Those who had been out of training for a longer period of time more often believed that they had received adequate exposure to peripheral nerve surgery during training.

There may be many ways in which to account for this finding. Older neurosurgeons may never have believed that complex peripheral nerve surgery was a large part of their practice and, therefore, deemed a less extensive experience adequate for their education. Furthermore, in the past a nihilistic, conservative, nonoperative approach was often espoused for the treatment of peripheral nerve problems. What may have been, in reality, a limited exposure to peripheral nerve problems was therefore seen as sufficient. Younger neurosurgeons, because they have had a more comprehensive education, may be more aware of the advances in peripheral nerve surgery and newer techniques, and may be more cognizant of having what was, in fact, limited exposure to this area during training. 


\section{A. Maniker and M. Passannante}

Despite reporting perceptions of inadequate exposure to peripheral nerve surgery during training, discomfort with many procedures, and few cases seen in practice, the overwhelming majority of respondents $(97.2 \%)$ believed that peripheral nerve surgery should remain part of the neurosurgical resident curriculum. Neurosurgeons remain committed to this important area of practice. A program of action to remedy the deficiencies uncovered by this survey would rightly be addressed at the highest levels of neurosurgical educational and organizational leadership.

This survey was intended as a tool for organized neurosurgery and neurosurgeons to examine their practice patterns and attitudes toward peripheral nerve surgery at the start of the 21st century. It may now serve as a useful starting point for a discussion regarding the future of peripheral nerve surgery and the neurosurgeon.

\section{Acknowledgments}

Special thanks to Mr. Nicholas Hlinka, Ms. Sandy Meyer, Ms. Pat
King, Dr. Peter Carmel, the Executive Committee of the Joint Section on Spine and Peripheral Nerve, and the Department of Neurosurgery at the New Jersey Medical School.

\section{References}

1. Cummings SM, Savitz LA, Konrad TR: Reported response rates to mailed physician questionnaires. Health Serv Res 35: 1347-1355, 2001

2. Kellerman SE, Herold J: Physician response to surveys. A review of the literature. Am J Prev Med 20:61-67, 2001

3. Kelly DL Jr: Now is our time. The 1991 AANS presidential address. J Neurosurg 75:677-684, 1991

Manuscript received September 10, 2002.

Accepted in final form February 10, 2003.

Address reprint requests to: Allen Maniker, M.D., Doctors Office Center, 90 Bergen Street, \#8th floor, Newark, New Jersey 07103. email: maniker@umdnj.edu. 